\title{
Performance Improvement for Port Loading and Unloading Process with Process Mining
}

\author{
Amanda Dewi Paramita ${ }^{1}$ and Iwan Vanany ${ }^{1}$
}

\begin{abstract}
Port is one of the important things in trade and logistics. The major influence factor of port productivity is the cycle time for loading and unloading. The container activity in port logistics consists of several activities, such as discharging, loading, receiving, delivery, gate-in, and gate-out, among others. These activities using various equipment including container cranes, Rubber Tyred Gantry (RTG), trucks, and other related machinery. The availability of equipment is one of the factors the influence delays in container activities, which can negatively affect productivity. The productivity of the port will affect to Key Performance Indicator (KPI) of the port. The purpose of this study is to analyze the flow of containers that used process mining. Moreover, it provides a recommendation to increase the productivity at Terminal Nilam Multipurpose Port of Tanjung Perak. Process mining method includes many of other analysis, that is discovery, prediction and real-time, bottlenecks and deviation analysis. Disco program used to analyze container activity, besides this, the disco program also use to determine bottleneck mapping that effect to port productivity. The result show delivery and receiving are the cause of the bottleneck. Delivery cancel is known as an activity that inappropriate with the ideal process and indicates as an error in the flow of container activities. The improvement scenario carried out from the results of the analysis obtained is to minimize the occurrence of cancel loading delivery, to obtain a time reduction of \pm 11.6 hours. This will affect the productivity of container activities.
\end{abstract}

Keywords-Cycle Time, Process Mining, Bottleneck, Loading, Receiving, and Delivery Cancel.

\section{INTRODUCTION}

The performance of a port is influenced by the cycle of loading and unloading activities, as is the case at the Terminal Nilam Multipurpose Port of Tanjung Perak, increasing from year to year in the cycle of loading and unloading activities, especially container loading and unloading. Container activities include discharging, loading, delivery, receiving, gate-in and gate-out. Whereas in carrying out container activities it is supported by tools and facilities including container cranes, Rubber Tyred Gantry (RTG), trucks, Reach Steaker (RS) and others. Based on observations in the field, several conditions that result in an increase in the loading and unloading cycle, from, among others, the existence of queues when the

\footnotetext{
${ }^{1}$ Amanda Dewi Paramita is with Department of Management Technology, Institut Teknologi Sepuluh Nopember, Indonesia. Email: amandadewiparamita@gmail.com.

${ }^{2}$ Iwan Vanany is with Department of Industrial Engineering, Institut Teknologi Sepuluh Nopember, Indonesia. Email: vanany@ie.its.ac.id.
}

trailer will load the container from the ship or vice versa. cause delays from a predetermined schedule and cause unwanted activity and impact on productivity achievement. Currently, the Terminal Nilam uses a spiner application to record each container loading and unloading activity. The results of the recording can be used as data to measure the productivity of a port which is one of the indicators in achieving KPI. So it is necessary to analyze loading and unloading performance using process mining, that is by using secondary data from the event log. By choosing this method, it can be known transparently the difference between ideal business processes and real processes that occur in the field.

\section{METHOD}

\section{A. Flow Chart}

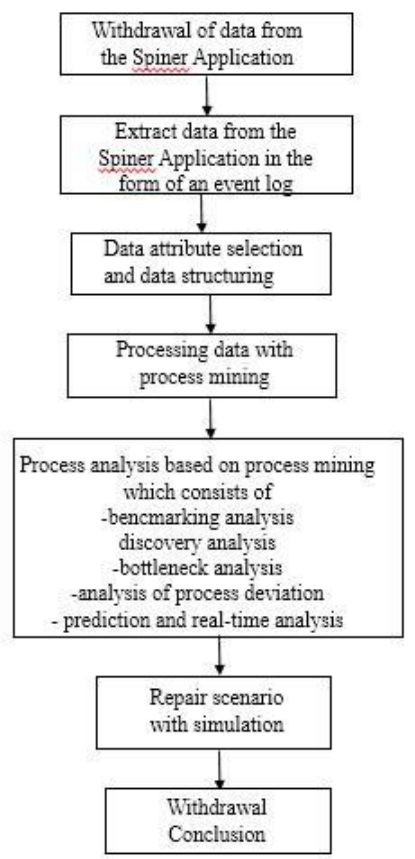

Figure 1. Flow chart of methodology

\section{B. Extracting}

Selecting and structuring data Extracting data is obtained from the export of the spiner application data. Event log data consists of various types of attributes, including date and time of loading and unloading activities, ship code, container size ( $20 \mathrm{ft}, 40 \mathrm{ft}$ ), container type (general purpose container, temperature, reefer container), goods activities 
(stabilizing, delivery, stacking), type of loading and unloading (loading, discharge), equipment used (container crane, RTG and truck), ship code, number of production and production units.

The next step is to select the data on the extraction results to determine the required attributes. This is because the extraction data obtained is very broad and not all of these attributes are following the data requirements needed to analyze the process. The data needed is data that has an identity, activity and time of operational activities. Next is doing the rearrangement of event log data by equating the format and compiling data according to the mapping of each activity related to the flow of the container activity process.

\section{Processing data with Process mining}

Process analysis to be carried out in the form of analysis of discovery, bottleneck, process deviations, and prediction and real-time, with the explanation as follows:

- Benchmarking analysis to identify each data in the event log, as needed.

- Discovery analysis to identify business process models and on which processes occur deviations and obtain the calculation of the average time of each activity process.

- Analysis of bottlenecks which can later be used as a reference for improving performance by knowing the influential factors.

- Analysis of process deviations that obtained differences between the flow of container processes that occur in the field with the ideal process.

- Prediction and real-time analysis with the results obtained can be predicted against the possibility of errors in the process that will occur and can be monitored in real time the flow of the container process so that it can be dealt with quickly and precisely if there is an error in a process.

\section{Scenario improvement with simulation}

The model of the simulation will be used to determine the best improvement scenario. And an indicator that will be used to determine the best repair scenario is that there is no bottleneck so that the time of container activities is faster with higher loading and unloading productivity results.

After obtaining several model simulations and variations, an analysis of several variants was obtained. By identifying several of these variants, several analyzes were obtained relating to the flow of business processes including bottleneck analysis, discovery and process deviations.

\section{E. Withdrawal conclusion}

Drawing conclusions is done after doing all the analyses carried out and obtained the most optimal results. After obtaining the results of the analysis, recommendations can be given to PT Pelindo III for further improvements.

\section{RESULTS AND DISCUSSION}

\section{A. Withdrawal of data from the Spiner Application}

The data used is in the form of event logs that are obtained from the export of data from spiner.

\section{B. The results of the process of selecting data attributes and structuring data}

After the event $\log$ is formed, then the data is selected and structured, this is because the extraction data obtained is very broad and not all of the attributes are following the data requirements for conducting process analysis. The data needed is data that has an identity, activity and time of operational activities. Next is to reorganize event log data by equating the format and compiling data according to the mapping of each activity related to the flow of the container activity process.

\section{Event log Information}

Initial conditions where $21,382 \operatorname{logs}$ are input into this model

TABLE 1.

EVENT LOG ANALYSIS

\begin{tabular}{ll}
\hline \hline \multicolumn{2}{c}{ Event log analysis } \\
\hline Number of case & 341 \\
Number of activity & 16 \\
Number of events & 21.382 \\
Start & 02.11 .2018 \\
& $2: 45: 00$ \\
End & 31.03 .2019 \\
& $16: 33: 00$ \\
Median case duration & 17,7 hours \\
Mean case duration & 65,4 hours \\
Number of variants & 279 \\
\hline \hline
\end{tabular}

\section{Statistical Information}

The following are statistical information including:

a. Case Duration: Shows information about the duration of the case contained in the model.

b. Activity: Shows the frequency of each loading and unloading activity consisting of loading, discharge, receiving and delivery

c. Resource: Indicates the statistics of the resource frequency used in each case. In this case, the intended resource is the loading and unloading equipment used, namely Container Crane.

\section{E. Process Analysis based on mining processes}

\section{1) Analysis of the benchmarking process}

in analyzing the benchmarking process classification is carried out on several parameters relating to the container loading and unloading activities, including resources, container size, container shipping destinations, shipping, and activity agent names. 
The $1^{\text {st }}$ International Conference on Business and Management of Technology (IConBMT)

TABLE 1.

EVENT LOG

\begin{tabular}{|c|c|c|c|c|c|c|c|c|c|c|c|c|}
\hline KD_CABANG & KD_TERMINAL & NO_APPROVAL & $\begin{array}{l}\text { UKURAN } \\
\text { PETIKEMAS }\end{array}$ & $\begin{array}{l}\text { KODE } \\
\text { ALAT }\end{array}$ & $\begin{array}{l}\text { SATUAN } \\
\text { PRODUKSI }\end{array}$ & $\begin{array}{c}\text { JUMLAH } \\
\text { PRODUKSI }\end{array}$ & NAMA_POL & NAMA_POD & AGENT_NAME & KEGIATAN_BRG & START_WORK & END_WORK \\
\hline 2 & 4 & SR020418000001 & 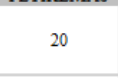 & $\mathrm{NCC} 04$ & BOX & 10 & MAKASSAR & MAKASSAR & $\begin{array}{c}\text { PELAYARAN } \\
\text { CARAKA TIRTA } \\
\text { PERKAS }\end{array}$ & $\begin{array}{c}\text { DELIVERY } \\
\text { LOSSING-DCL }\end{array}$ & $11 / 2 / 20182: 45$ & 11/2/2018 16:55 \\
\hline 2 & 4 & SR020418000001 & 40 & $\mathrm{NCCO4}$ & BOX & 10 & MAKASSAR & MAKASSAR & $\begin{array}{l}\text { PELAYARAN } \\
\text { CARAKA TIRTA } \\
\text { PERKAS }\end{array}$ & $\begin{array}{c}\text { DELIVERY } \\
\text { LOSSING-DCL }\end{array}$ & $11 / 2 / 20182: 45$ & $11 / 22201816: 55$ \\
\hline 2 & 4 & SR020418000001 & 20 & $\mathrm{NCCO4}$ & BOX & 10 & MAKASSAR & MAKASSAR & $\begin{array}{l}\text { PELAYARAN } \\
\text { CARAKA TIRTA } \\
\text { PERKAS }\end{array}$ & $\begin{array}{c}\text { DELIVERY } \\
\text { LOSSING-DCL }\end{array}$ & $11 / 2 / 20182: 45$ & $11 / 2 / 201816: 55$ \\
\hline 2 & 4 & SR020418000001 & 40 & $\mathrm{NCCO4}$ & BOX & 8 & MAKASSAR & MAKASSAR & $\begin{array}{l}\text { PELAYARAN } \\
\text { CARAKA TIRTA } \\
\text { PERKAS }\end{array}$ & $\begin{array}{c}\text { DELIVERY } \\
\text { LOSSING-DCL }\end{array}$ & $11 / 2 / 20182: 45$ & $11 / 2 / 201816: 55$ \\
\hline 2 & 4 & SR020418000001 & 40 & $\mathrm{NCCO4}$ & BOX & 8 & MAKASSAR & MAKASSAR & $\begin{array}{c}\text { PELAYARAN } \\
\text { CARAKA TIRTA } \\
\text { PERKAS }\end{array}$ & $\begin{array}{c}\text { DELIVERY } \\
\text { LOSSING-DCL }\end{array}$ & $11 / 2 / 20182: 45$ & $11 / 2 / 201816: 55$ \\
\hline 2 & 4 & SR020418000001 & 20 & $\mathrm{NCCO4}$ & BOX & 122 & MAKASSAR & MAKASSAR & $\begin{array}{l}\text { PELAYARAN } \\
\text { CARAKA TIRTA } \\
\text { PERKAS }\end{array}$ & $\begin{array}{c}\text { DELIVERY } \\
\text { LOSSING-DCL }\end{array}$ & $11 / 2 / 20182: 45$ & 11/2/2018 16:55 \\
\hline 2 & 4 & SR020418000001 & 20 & $\mathrm{NCC} 04$ & BOX & 122 & MAKASSAR & MAKASSAR & $\begin{array}{l}\text { PELAYARAN } \\
\text { CARAKA TIRTA } \\
\text { PERKAS }\end{array}$ & $\begin{array}{c}\text { DELIVERY } \\
\text { LOSSING-DCL }\end{array}$ & $11 / 2 / 20182: 45$ & 11/2/2018 16:55 \\
\hline 2 & 4 & SR020418000001 & 40 & $\mathrm{NCCO4}$ & BOX & 4 & MAKASSAR & MAKASSAR & $\begin{array}{c}\text { PELAYARAN } \\
\text { CARAKA TIRTA } \\
\text { PERKAS }\end{array}$ & $\begin{array}{c}\text { DELIVERY } \\
\text { LOSSING-DCL }\end{array}$ & $11 / 2 / 20182: 45$ & 11/2/2018 16:55 \\
\hline 2 & 4 & SR020418000001 & 40 & $\mathrm{NCC} 04$ & BOX & 10 & MAKASSAR & MAKASSAR & $\begin{array}{c}\text { PELAYARAN } \\
\text { CARAKA TIRTA } \\
\text { PERKAS }\end{array}$ & $\begin{array}{c}\text { DELIVERY } \\
\text { LOSSING-DCL }\end{array}$ & $11 / 2 / 20182: 45$ & $11 / 2 / 201816: 55$ \\
\hline 2 & 4 & SR020418000001 & 40 & $\mathrm{NCCO4}$ & BOX & 4 & MAKASSAR & MAKASSAR & $\begin{array}{l}\text { PELAYARAN } \\
\text { CARAKA TIRTA } \\
\text { PERKAS }\end{array}$ & $\begin{array}{c}\text { DELIVERY } \\
\text { LOSSING-DCL }\end{array}$ & $11 / 2 / 20182: 45$ & $11 / 2 / 201816: 55$ \\
\hline 2 & 4 & SR020418000107 & 20 & $\mathrm{NCCO} 3$ & BOX & 1 & $\begin{array}{l}\text { TANJUNG } \\
\text { PERAK }\end{array}$ & $\begin{array}{c}\text { BALIKPAPA } \\
\mathrm{N}\end{array}$ & $\begin{array}{l}\text { TANTO KARYA } \\
\text { UTAMA, PT }\end{array}$ & $\begin{array}{c}\text { DELIVERY } \\
\text { LOSSING-DCL }\end{array}$ & $11 / 3 / 20182: 45$ & 11/3/2018 12:11 \\
\hline 2 & 4 & SR020418000107 & 40 & $\mathrm{NCC} 03$ & Box & 1 & $\begin{array}{l}\text { TANJUNG } \\
\text { PERAK }\end{array}$ & $\begin{array}{c}\text { BALIKPAPA } \\
\mathrm{N}\end{array}$ & $\begin{array}{l}\text { TANTO KARYA } \\
\text { UTAMA, PT }\end{array}$ & $\begin{array}{c}\text { DELIVERY } \\
\text { LOSSING-DCL }\end{array}$ & $11 / 3 / 20182: 45$ & 11/3/2018 12:11 \\
\hline 2 & 4 & SR020418000107 & 20 & $\mathrm{NCC} 03$ & BOX & 9 & $\begin{array}{l}\text { TANJUNG } \\
\text { PERAK }\end{array}$ & $\begin{array}{c}\text { BALIKPAPA } \\
\mathrm{N}\end{array}$ & $\begin{array}{l}\text { TANTO KARYA } \\
\text { UTAMA, PT }\end{array}$ & $\begin{array}{c}\text { DELIVERY } \\
\text { LOSSING-DCL }\end{array}$ & $11 / 3 / 20182: 45$ & 11/3/2018 12:11 \\
\hline 2 & 4 & SR020418000107 & 20 & $\mathrm{NCCO} 3$ & BOX & 1 & $\begin{array}{l}\text { TANJUNG } \\
\text { PERAK }\end{array}$ & $\begin{array}{c}\text { BALIKPAPA } \\
\mathrm{N}\end{array}$ & $\begin{array}{l}\text { TANTO KARYA } \\
\text { UTAMA, PT }\end{array}$ & $\begin{array}{c}\text { DELIVERY } \\
\text { LOSSING-DCL }\end{array}$ & $11 / 3 / 20182: 45$ & 11/3/2018 12:11 \\
\hline 2 & 4 & SR020418000107 & 20 & $\mathrm{NCCO} 3$ & BOX & 9 & $\begin{array}{l}\text { TANJUNG } \\
\text { PERAK }\end{array}$ & $\begin{array}{c}\text { BALIKPAPA } \\
\mathrm{N}\end{array}$ & $\begin{array}{c}\text { TANTO KARYA } \\
\text { UTAMA, PT }\end{array}$ & $\begin{array}{c}\text { DELIVERY } \\
\text { LOSSING-DCL }\end{array}$ & $11 / 3 / 20182: 45$ & 11/3/2018 12:11 \\
\hline 2 & 4 & SR020418000107 & 40 & $\mathrm{NCCO} 3$ & BOX & 1 & $\begin{array}{l}\text { TANJUNG } \\
\text { PERAK }\end{array}$ & $\begin{array}{c}\text { BALIKPAPA } \\
\mathrm{N}\end{array}$ & $\begin{array}{c}\text { TANTO KARYA } \\
\text { UTAMA, PT }\end{array}$ & $\begin{array}{c}\text { DELIVERY } \\
\text { LOSSING-DCL }\end{array}$ & $11 / 3 / 20182: 45$ & 11/3/2018 12:11 \\
\hline 2 & 4 & SR020418000107 & 20 & $\mathrm{NCCO} 3$ & BOX & 38 & $\begin{array}{l}\text { TANJUNG } \\
\text { PERAK }\end{array}$ & $\begin{array}{c}\text { BALIKPAPA } \\
\mathrm{N}\end{array}$ & $\begin{array}{l}\text { TANTO KARYA } \\
\text { UTAMA, PT }\end{array}$ & $\begin{array}{c}\text { DELIVERY } \\
\text { LOSSING-DCL }\end{array}$ & $11 / 3 / 20182: 45$ & 11/3/2018 12:11 \\
\hline 2 & 4 & SR020418000107 & 20 & $\mathrm{NCC} 03$ & BOX & 38 & $\begin{array}{l}\text { TANJUNG } \\
\text { PERAK }\end{array}$ & $\begin{array}{c}\text { BALIKPAPA } \\
\mathrm{N}\end{array}$ & $\begin{array}{l}\text { TANTO KARYA } \\
\text { UTAMA, PT }\end{array}$ & $\begin{array}{c}\text { DELIVERY } \\
\text { LOSSING-DCL }\end{array}$ & $11 / 3 / 20182: 45$ & $11 / 3 / 201812: 11$ \\
\hline 2 & 4 & SR020418000002 & 20 & $\mathrm{NCC} 05$ & BOX & 10 & $\begin{array}{c}\text { SAMARIND } \\
\text { A }\end{array}$ & SAMARINDA & $\begin{array}{l}\text { SALAM PACIFIC } \\
\text { INDONESIA } \\
\text { LINES, PT }\end{array}$ & DISCHARGE-DCH & 11/3/2018 12:10 & $11 / 4 / 20186: 13$ \\
\hline 2 & 4 & SR020418000002 & 40 & $\mathrm{NCC} 05$ & BOX & 9 & $\begin{array}{c}\text { SAMARIND } \\
\text { A }\end{array}$ & SAMARINDA & $\begin{array}{l}\text { SALAM PACIFIC } \\
\text { INDONESIA } \\
\text { LINES, PT }\end{array}$ & DELIVERY-DLV & $11 / 3 / 201812: 10$ & $11 / 4 / 20186: 13$ \\
\hline 2 & 4 & SR020418000002 & 40 & $\mathrm{NCC} 05$ & BOX & 5 & $\begin{array}{c}\text { SAMARIND } \\
\text { A }\end{array}$ & SAMARINDA & $\begin{array}{l}\text { SALAM PACIFIC } \\
\text { INDONESIA } \\
\text { LINES, PT }\end{array}$ & $\begin{array}{c}\text { DELIVERY } \\
\text { LOSSING-DCL }\end{array}$ & $11 / 3 / 2018$ 12:10 & $11 / 4 / 20186: 13$ \\
\hline 2 & 4 & SR020418000002 & 40 & $\mathrm{NCC} 05$ & BOX & 9 & $\begin{array}{c}\text { SAMARIND } \\
\text { A }\end{array}$ & SAMARINDA & $\begin{array}{l}\text { SALAM PACIFIC } \\
\text { INDONESIA } \\
\text { LINES, PT }\end{array}$ & DISCHARGE-DCH & 11/3/2018 12:10 & $11 / 4 / 20186: 13$ \\
\hline
\end{tabular}

TABLE 2.

THE Results OF THE BENCHMARK PROCESS ANALYSIS FOR "RESOURCES"

\begin{tabular}{ll}
\hline \hline Resources Name & Procentage \\
\hline CC04 & $4485(20,98 \%)$ \\
CC05 & $4335(20,27 \%)$ \\
CC03 & $3903(18,25 \%)$ \\
CC01 & $3237(15,14 \%)$ \\
RTG 01 & $1154(5,4 \%)$ \\
RTG 04 & $1049(4,91 \%)$ \\
RTG 05 & $917(4,29 \%)$ \\
RTG 03 & $754(3,53 \%)$ \\
RTG 02 & $740(3,46 \%)$ \\
RTG 06 & $638(2,98 \%)$ \\
RS 01 & $166(0,78 \%)$ \\
SHIP 01 & $4(0,02 \%)$ \\
\hline \hline
\end{tabular}

TABLE 3.

MEAN DURATION FOR RESOURCES

\begin{tabular}{cc}
\hline \hline Resources Name & Mean duration \\
\hline CC 04” & 21 hour 36 minutes \\
CC 05 & 22 hours 2 minutes \\
CC 03 & 19 hours 46 minutes \\
CC 01 & 22 hours 23 minutes \\
RTG 01 & 20 hours 23 minutes \\
RTG 04 & 21 hours 36 minutes \\
RTG 05 & 21 hours 40 minutes \\
RTG 03 & 22 hours 13 minutes \\
RTG 02 & 19 hours 50 minutes \\
RTG 06 & 21 hours 14 minutes \\
RTG 06 & 17 hours 17 minutes \\
RTG 06 & 21 hours 38 minutes \\
\hline \hline
\end{tabular}


The $1^{\text {st }}$ International Conference on Business and Management of Technology (IConBMT)

August 3rd 2019, Institut Teknologi Sepuluh Nopember, Surabaya, Indonesia

From the results of the analysis of the benchmark process for these resources, it can be seen that the highest level of tool used is the use of $\mathrm{CC} 04$ with a percentage value of $20.98 \%$, with an average duration of use for one loading and unloading cycle of 21 hour 36 minutes.

TABLE 4.

The Results of The Benchmark Process AnAlysis FOR "Container

\begin{tabular}{ll}
\multicolumn{2}{c}{ SIZE" } \\
\hline \hline Type of containers & $\mathbf{2}$ type \\
\hline Frequency "size $\mathbf{2 0} \mathbf{f t}$ " & $13.175(61,62 \%)$ \\
Frequency "size 40 ft" & $8.207(38,38 \%)$ \\
\hline \hline
\end{tabular}

From the analysis of the benchmark process for the size of the container, it can be seen that the highest level of container usage is the use of containers measuring $20 \mathrm{ft}$ with a percentage of $61.62 \%$.

TABLE 5.

The Results of THe Benchmark Process ANALysis For "DELIVERY DESTINATION"

\begin{tabular}{lc}
\hline \hline Delivery destination & Frequency \\
\hline to Samarinda & $6442(30,13 \%)$ \\
to Makassar & $3254(15,22 \%)$ \\
to Kupang & $2264(10,59 \%)$ \\
to Tarakan & $1987(9,29 \%)$ \\
to Balikpapan & $1502(7,02 \%)$ \\
to Ambon & $1490(6,97 \%)$ \\
to Pantoloan & $996(4,66 \%)$ \\
to Palaran & $827(3,87 \%)$ \\
to Nunukan & $714(3,34 \%)$ \\
to Timika & $541(2,53 \%)$ \\
to Banjarmasin & $521(2,44 \%)$ \\
to Merauke & $364(1,7 \%)$ \\
to Dobo & $151(0,71 \%)$ \\
to Bau Bau & $97(0,45 \%)$ \\
to Jakarta & $87(0,41 \%)$ \\
to Kendari & $57(0,27 \%)$ \\
to Tual & $50(0,23 \%)$ \\
to Maumere & $20(0,09 \%)$ \\
to Ampenan & $18(0,08 \%)$ \\
\hline \hline
\end{tabular}

From the results of the analysis of the benchmark process for delivery, it can be seen that the most shipping destination is shipping to Samarinda with a percentage value of $30.13 \%$.

From the results of the analysis of the process, it can be seen that the most loading and unloading activities are loading activities with a frequency of 7731 times with an average duration of 20 hours 52 minutes.

From the results of the analysis of the process, it can be seen that shipping agents that often carry out container loading and unloading activities are PT Meratus Line with Frequency of 9105 times with a percentage of $42.58 \%$.
TABLE 6.

THE RESULTS OF THE BENCHMARK PROCESS ANALYSISFOR “ACTIVITY”

\begin{tabular}{ll}
\hline \hline \multicolumn{1}{c}{ Activity } & \multicolumn{1}{c}{ Procentage } \\
\hline Delivery & $7731(36,16 \%)$ \\
Delivery cancel loading & $83(2,98 \%)$ \\
Delivery lossing & $2582(12,08 \%)$ \\
Delivery lossing transhipment direct between terminal & $2(0,01 \%)$ \\
Delivery lossing transhipment transit CY & $130(0,61 \%)$ \\
Discharge & $4320(20,2 \%)$ \\
Loading & $7731(36,16 \%)$ \\
Loading transhipment between terminal & $300(1,4 \%)$ \\
Receiving & $4882(22,83 \%)$ \\
Receiving lossing & $16(0,07 \%)$ \\
Receiving tambah & $14(0,07 \%)$ \\
Reefer” & $1(0 \%)$ \\
Relocation haulage & $120(0,56 \%)$ \\
Shifting with landing & $9(0,04 \%)$ \\
Shifting with landing CY & $3(0,01 \%)$ \\
Shifting without landing & $6(0,03 \%)$ \\
\hline \hline
\end{tabular}

TABLE 7.

MEAN DURATION FOR ACTIVITY

\begin{tabular}{ll}
\hline \hline \multicolumn{1}{c}{ Activity } & \multicolumn{1}{c}{ Mean duration } \\
\hline Delivery & 23 hours 51 seconds \\
Delivery cancel loading & 1 hour 3 hours \\
Delivery lossing & 21 hours 51 minutes \\
Delivery lossing transhipment direct & 22 hours 39 minutes \\
between terminal” & \\
Delivery lossing transhipment transit CY & 1 hour 3 hours \\
Discharge & 21 hour 50 minutes \\
Loading & 20 hours 52 minutes \\
Loading transhipment antar terminal & 1 hour 2 hours \\
Receiving & 20 hours 29 minutes \\
Receiving lossing & 1 hour 13 minutes \\
Receiving tambah & 1 hour 11 hour \\
Reefer & 1 hour 1 hour \\
Relocation haulage & 22 hours 51 minutes \\
Shifting with landing & 1 hour 3 hours \\
Shifting with landing CY & 1 day 1 hour \\
Shifting without landing” & 23 hours 32 minutes \\
\hline \hline
\end{tabular}

TABLE 8.

THE RESULTS OF THE BENCHMARK PROCESS ANALYSISFOR "SHIPPING AGENT"

\begin{tabular}{ll}
\hline \hline \multicolumn{1}{c}{ Name of shipping agent } & \multicolumn{1}{c}{ Procentage } \\
\hline PT Meratus Line & $9105(42,58 \%)$ \\
PT SPIL & $7524(35,19 \%)$ \\
PT Tanto Karya Utama & $2338(10,93 \%)$ \\
PT Perusahaan Pelayaran Nusantara Panurjawan & $1448(6,77 \%)$ \\
PT Pelayaran Caraka Tirta Perkasa" & $967(4,52 \%)$ \\
\hline \hline
\end{tabular}




\section{2) Analysis of process discovery}

In analyzing the discovery process, 3 (three) variants can be found with the highest ranking with the following details:

\begin{tabular}{lll}
\multicolumn{3}{c}{ TABLE 9. } \\
TOP 3 VARIANT \\
\hline \hline Top 3 variant & case & Event \\
\hline Variant 1 & 15 & 30 \\
Variant 2 & 12 & 12 \\
Variant 3 & 8 & 8 \\
Total & 35 & \\
\hline \hline
\end{tabular}

From Table 9. it can be seen that there are 3 paths most often carried out in the process of loading and unloading activities. The 3 lines cover $10.26 \%$ of the 341 trajectories that occur in loading and unloading activities.

\begin{tabular}{|c|c|c|c|}
\hline No Case & Activity & Resource & Duration \\
\hline \multirow[t]{2}{*}{ Case 1} & Delivery & RTG 01 & 17 hours 17 minutes \\
\hline & Delivery & RTG 01 & 19 hours 17 minutes \\
\hline \multirow[t]{2}{*}{ Case 2} & Delivery & RTG 01 & 17 hours 17 minutes \\
\hline & Delivery & RTG 01 & 17 hours 17 minutes \\
\hline \multirow[t]{2}{*}{ Case 3} & Delivery & RTG 01 & 17 hours 17 minutes \\
\hline & Delivery & RTG 01 & 17 hours 17 minutes \\
\hline \multirow[t]{2}{*}{ Case 4} & Delivery & RTG 04 & 19 hours 17 minutes \\
\hline & Delivery & RTG 04 & 19 hours 17 minutes \\
\hline \multirow[t]{2}{*}{ Case 5} & Delivery & RTG 01 & 19 hours 17 minutes \\
\hline & Delivery & RTG 01 & 19 hours 17 minutes \\
\hline \multirow[t]{2}{*}{ Case 6} & Delivery & RTG 01 & 17 hours \\
\hline & Delivery & RTG 01 & 17 hours \\
\hline \multirow[t]{2}{*}{ Case 7} & Delivery & RTG 04 & 1 hour 34 minutes \\
\hline & Delivery & RTG 04 & 1 hour 34 minutes \\
\hline \multirow[t]{2}{*}{ Case 8} & Delivery & RTG 04 & 17 hours 19 minutes \\
\hline & Delivery & RTG 04 & 17 hours 19 minutes \\
\hline \multirow[t]{2}{*}{ Case 9} & Delivery & RTG 01 & 17 hours 42 minutes \\
\hline & Delivery & RTG 01 & 17 hours 42 minutes \\
\hline \multirow[t]{2}{*}{ Case 10} & Delivery & RTG 05 & 19 hours 58 minutes \\
\hline & Delivery & RTG 05 & 19 hours 58 minutes \\
\hline \multirow[t]{2}{*}{ Case 11} & Delivery & RTG 05 & 1 hour 7 hours \\
\hline & Delivery & RTG 05 & 1 hour 7 hours \\
\hline \multirow[t]{2}{*}{ Case 12} & Delivery & RTG 05 & 17 hours 25 minutes \\
\hline & Delivery & RTG 01 & 17 hours 25 minutes \\
\hline \multirow[t]{2}{*}{ Case 13} & Delivery & RTG 01 & 22 hours 5 minutes \\
\hline & Delivery & RTG 01 & 19 hours 26 minutes \\
\hline \multirow[t]{2}{*}{ Case 14} & Delivery & RTG 02 & 9 hours 50 minutes \\
\hline & Delivery & RTG 02 & 1 hour 4 hours \\
\hline \multirow[t]{3}{*}{ Case 15} & Delivery & RTG 01 & 17 hours \\
\hline & Delivery & RTG 01 & 17 hours \\
\hline & \multicolumn{2}{|c|}{ Mean duration } & 19 hours 32 minutes \\
\hline
\end{tabular}

TABLE 11.

VARIANT 2

\begin{tabular}{|c|c|c|c|}
\hline No Case & Activity & Resource & Duration \\
\hline Case 1 & Delivery & RTG 04 & 17 hours 17 minutes \\
\hline Case 2 & Delivery & RTG 01 & 20 hours 13 minutes \\
\hline Case 3 & Delivery & RTG 01 & 1 hour 11 hour \\
\hline Case 4 & Delivery & RTG 01 & 17 hours 19 minutes \\
\hline Case 5 & Delivery & RTG 01 & 11 hour 44 minutes \\
\hline Case 6 & Delivery & RTG 01 & 20 hours 3 minutes \\
\hline Case 7 & Delivery & RTG 05 & 1 hour 7 hours \\
\hline Case 8 & Delivery & RTG 05 & 13 hours 7 minutes \\
\hline Case 9 & Delivery & RTG 05 & 17 hours 25 minutes \\
\hline Case 10 & Delivery & RTG 01 & 8 hours 30 minutes \\
\hline Case 11 & Delivery & RTG 01 & 1 hour 5 hours \\
\hline \multirow[t]{2}{*}{ Case 12} & Delivery & RTG 01 & 1 hour 21 hour \\
\hline & \multicolumn{2}{|c|}{ Mean duration } & 22 hours 5 minutes \\
\hline
\end{tabular}

TABLE 12.

VARIANT 3

\begin{tabular}{|c|c|c|c|}
\hline No Case & Activity & Resource & Duration \\
\hline Case 1 & $\begin{array}{l}\text { Delivery cancel } \\
\text { loading }\end{array}$ & RTG 01 & 2 hours 7 minutes \\
\hline Case 2 & $\begin{array}{l}\text { Delivery cancel } \\
\text { loading }\end{array}$ & RTG 06 & 1 hour 7 minutes \\
\hline Case 3 & $\begin{array}{l}\text { Delivery cancel } \\
\text { loading }\end{array}$ & RTG 04 & 7 hours 50 minutes \\
\hline Case 4 & $\begin{array}{l}\text { Delivery cancel } \\
\text { loading }\end{array}$ & RTG 01 & 17 hours 45 minutes \\
\hline Case 5 & $\begin{array}{l}\text { Delivery cancel } \\
\text { loading }\end{array}$ & RTG 06 & 17 hours 2 minutes \\
\hline Case 6 & $\begin{array}{l}\text { Delivery cancel } \\
\text { loading }\end{array}$ & RTG 05 & 1 hour 29 minutes \\
\hline Case 7 & $\begin{array}{l}\text { Delivery cancel } \\
\text { loading }\end{array}$ & RTG 05 & 2 hours 12 minutes \\
\hline Case 8 & $\begin{array}{l}\text { Delivery cancel } \\
\text { loading }\end{array}$ & RTG 01 & 18 hours 33 minutes \\
\hline \multicolumn{3}{|c|}{ Mean duration } & 1 day 3 hours 30 minutes \\
\hline
\end{tabular}

From the discovery process analysis, by taking the top 3 variants, there were 2 emerging activities, namely delivery and delivery canceling activities, with an average duration of variants 1 for 19 hours 32 minutes, variant 2 for 22 hours 5 minutes, variant 3 for 3 hours 30 minutes. Thus it can be concluded that the highest duration of time is in variant 3 with activities in it, namely, cancel loading delivery. This indicates that the emergence of cancel loading delivery activities is one of the factors that cause the duration of the container loading and unloading cycle. From the table it can be seen that there are 3 paths most often carried out in the process of loading and unloading activities. The 3 lines cover $10.26 \%$ of the 341 trajectories that occur in loading and unloading activities.

3) Analysis of process bottleneck

Several factors that become bottleneck boosters can be identified by searching for process loops on the process 
The $1^{\text {st }}$ International Conference on Business and Management of Technology (IConBMT)

map. The loop process is expected to show the normal function of the process and can also show errors or process problems.

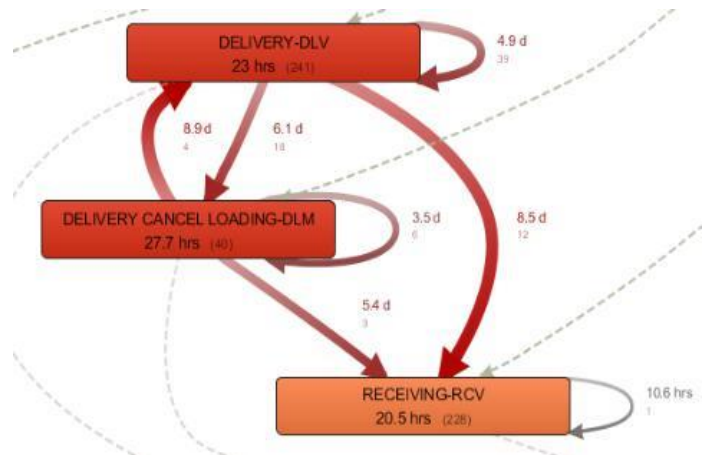

Figure 4. Loop Process

In Figure 4. it can be seen that activities in the red box are delivery, delivery cancel loading and receiving activities and it can be concluded that the three activities are bottlenecks in container activities.

TABLE 13.

RESUltS OF ANALYSIS OF THE BOTTLENECK PROCESS

\begin{tabular}{|c|c|c|c|}
\hline Activity & $\begin{array}{c}\text { Mean } \\
\text { dưfation }\end{array}$ & Min duration & Max duration \\
\hline Delivery & $23 \mathrm{jam}$ & $4,9 \mathrm{jam}$ & 7 hari -1 \\
\hline Delivery $\rightarrow$ delivery & 4,9 hari & 95 menit & 28,4 hari \\
\hline Delivery $\rightarrow$ Delivery cancel loading & 6,1 hari & 30,4 jam & 14,6 hari \\
\hline Delivery cancel loading & $27,7 \mathrm{jam}$ & $6,4 \mathrm{jam}$ & 4,9 hari $=: ?$ \\
\hline Delivery cancel loading $\rightarrow$ delivery & 8,9 hari & $3,1 \mathrm{jam}$ & 21,6 hari \\
\hline $\begin{array}{l}\text { Delivery cancel loading } \rightarrow \text { Delivery } \\
\text { cancel loading }\end{array}$ & 3,5 hari & $20,7 \mathrm{jam}$ & 7,2 hari \\
\hline Delivery $\rightarrow$ receiving & 8,5 hari & 30,4 jam & 16,2 hari \\
\hline Delivery cancel loading $\rightarrow$ receiving & 5,4 hari & $25,5 \mathrm{jam}$ & 7,2 hari \\
\hline Keceiving - & $20,5 \mathrm{jam}$ & $4,8 \mathrm{jam}$ & 7 hari $=:$ \\
\hline Receiving-receiving & $10,6 \mathrm{jam}$ & $10,6 \mathrm{jam}$ & $10,6 \mathrm{jam}$ \\
\hline
\end{tabular}

From the analysis of the bottleneck process, there were 3 activities which were the causes of a bottleneck, namely the activities of delivery, delivery cancel loading and receiving. From each of these activities which have the highest average duration is cancel loading delivery activities with an average duration of 27.7 hours.

4) Analysis of process deviations

In analyzing the deviations process, several processes can be found that do not match the actual process flow.

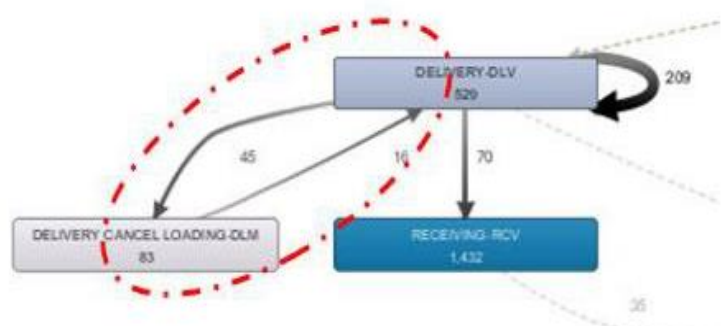

Figure 5. Analysis of Process Deviations

From Figure 5. it can be seen that there is a process that is not in accordance with the actual process, that is, after the delivery process occurs a cancel loading delivery process, which means that the delivery process is canceled due to some technical errors including job orders that do not meet the requirements it is not permitted to carry out the delivery process or it can be said that one of the processes on the job order request for the delivery process has passed.

\section{5) Analysis of process prediction and real time}

After analysis, it can be predicted that one of the errors in the container process flow is the emergence of cancel delivery activities because according to the actual container flow the process is greatly avoided because it will hinder other operational activities. If this activity occurs, there will be a buildup density in the stacking field, so that it will disrupt the smooth delivery of the next delivery activity.

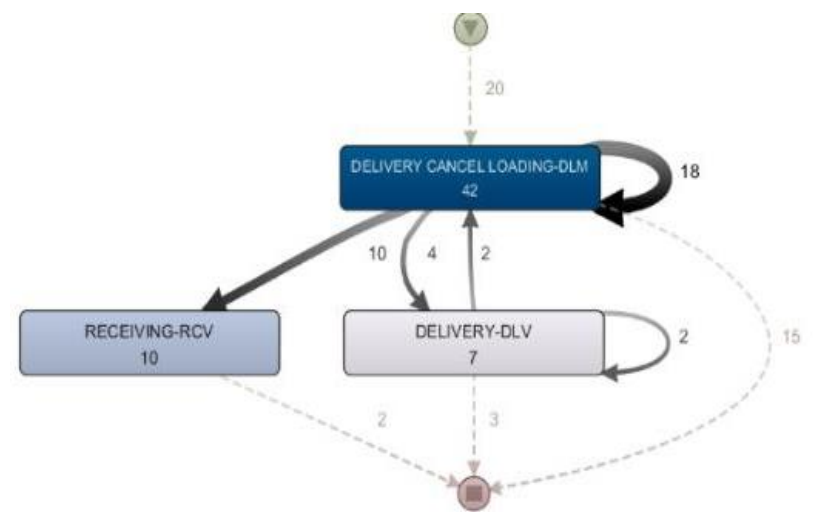

Figure 6. Prediction and Real Time Process

TABLE 14.

Results of ANALysis of The PREDiction AND REAL Time Process

\begin{tabular}{lc}
\hline \multicolumn{2}{l}{ Activity performance of delivery cancel loading } \\
\hline Total Duration & 45,1 hour \\
Mean duration & 25,8 hours \\
Max Duration & 60,7 hours \\
Min Duration & 7,8 hours \\
\hline \hline
\end{tabular}

$F$. Scenario improvements with simulation

1) Scenario improvement results from the analysis of the bottleneck process.

It Will be repaired for the delivery and receiving process flow. The improvement plan for the delivery process is to combine the print job order and gate in delivery as shown in Figure. 6. Meanwhile, for the receiving activity, repairs will be carried out by merging the print job order and gate in receiving as shown in Figure. 7. This is done to save the container cycle time so that it becomes more effective and efficient. 
The $1^{\text {st }}$ International Conference on Business and Management of Technology (IConBMT)

August 3rd 2019, Institut Teknologi Sepuluh Nopember, Surabaya, Indonesia

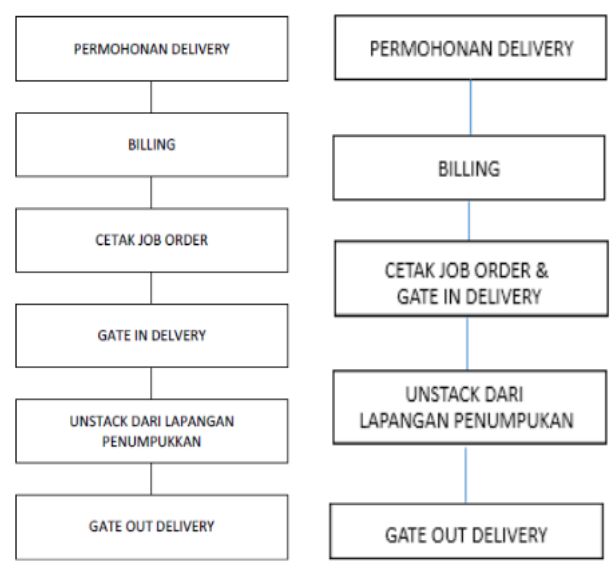

Figure 7. Delivery Activity - Existing (Left), the Result (Right)

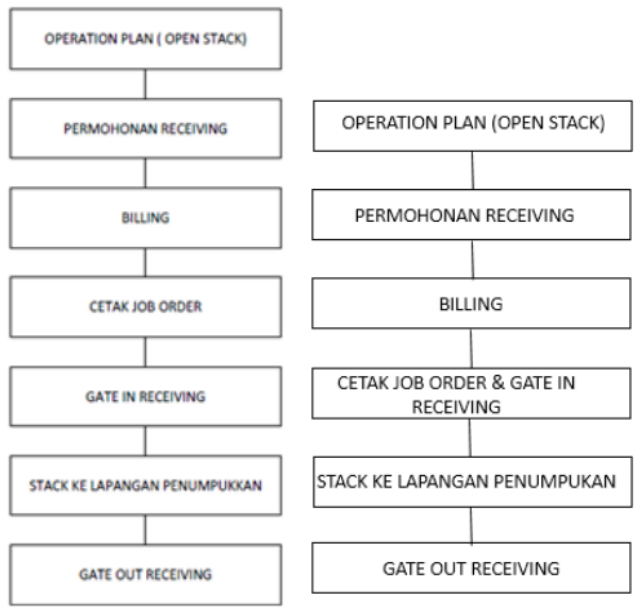

Figure 8. Receiving activity - Existing (left), the result (right)

2) Scenario improvement results from the discovery process and prediction and real-time process one improvement technique is to add a тепи in the display of the Order

Delivery Job, which displays the requirements for completing documents so that if one of the requirements cannot be met, the delivery request is automatically not approved.

If the cancel delivery process is omitted in the data, different simulations are obtained, with different variant and case results.

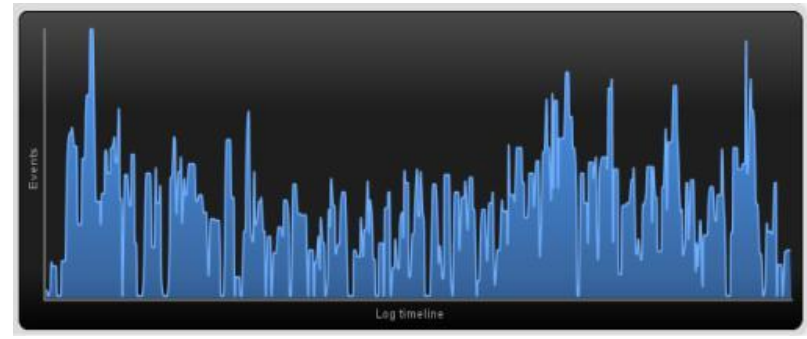

Figure 9. The Graph of the Analysis of Delivery Cancel Conditions is Omitted.
TABLE 15.

EVENT LOG ANALYSIS

\begin{tabular}{ll}
\hline \hline Number of case & 327 \\
Number of activity & 15 \\
Number of events & 21.299 \\
Start & 02.11 .2018 02:45:00 \\
End & 31.03 .2019 16:33:00 \\
Median case duration & 17,4 hours \\
Mean case duration & 53,8 hours \\
Number of variants & 255 \\
\hline \hline
\end{tabular}

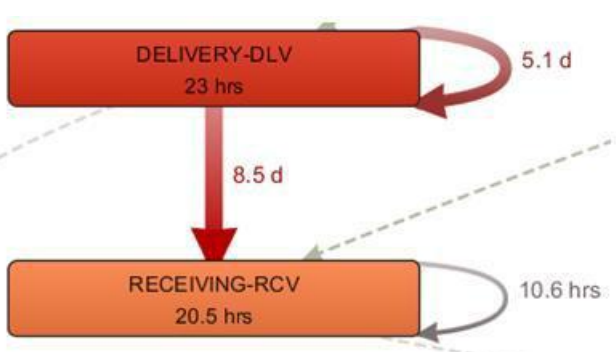

Figure 10. Loop process of delivery cancel conditions are omitted

When an improvement simulation is carried out by eliminating the cancel loading delivery, the results of the analysis of the activation process are as follows.

TABLE 16.

RESULTS OF PROCESS ANALYSIS FOR ACTIVITY

\begin{tabular}{cc}
\hline \hline Activity & Procentage \\
\hline Loading & $7731(36,3 \%)$ \\
Receiving & $4882(20,28 \%)$ \\
Discharge & $4320(10,93 \%)$ \\
Delivery & $1183(6,77 \%)$ \\
\hline \hline
\end{tabular}

TABLE 17.

MEAN DURATION FOR ACTIVITY

\begin{tabular}{cc}
\hline \hline Activity & Mean duration \\
\hline Loading & 20 hours 52 minutes \\
Receiving & 20 hours 29 minutes \\
Discharge & 21 hour 50 minutes \\
Delivery & 23 hours 51 seconds \\
\hline
\end{tabular}

ACTIVITY FREQUENCY AFTER DELETED DELIVERY CANCEL LOADING

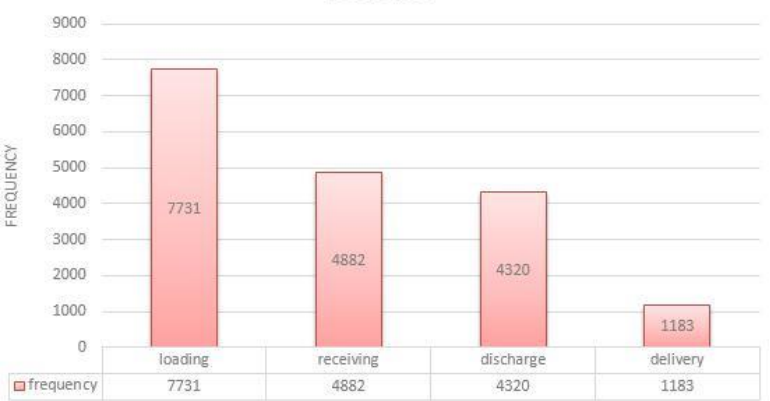

Figure.11. Activity Frequency After Deleted Delivery Cancel Loading. 
The $1^{\text {st }}$ International Conference on Business and Management of Technology (IConBMT)

August 3rd 2019, Institut Teknologi Sepuluh Nopember, Surabaya, Indonesia

TABLE 18. RESUltS OF PROCESS ANALYSIS FOR "RESOURCES"

\begin{tabular}{cc}
\hline \hline Resources & Procentage \\
\hline $\mathrm{CC} 04$ & $4485(21,06 \%)$ \\
$\mathrm{CC} 05$ & $4335(20,35 \%)$ \\
$\mathrm{CC} 03$ & $3903(18,32 \%)$ \\
$\mathrm{CC} 01$ & $3237(15,2 \%)$ \\
\hline \hline
\end{tabular}

TABLE 19.

\begin{tabular}{cl}
\multicolumn{2}{c}{ MEAN DURATION FOR RESOURCES } \\
\hline \hline Resources & Mean duration \\
\hline $\mathrm{CC} 04$ & 21 hour 36 minutes \\
$\mathrm{CC} 05$ & 22 hours 32 minutes \\
$\mathrm{CC} 03$ & 19 hours 46 minutes \\
$\mathrm{CC} 01$ & 22 hours 5minutes \\
\hline \hline
\end{tabular}

RESOURCE FREQUENCY AFTER DELETED DELIVERY CANCEL LOADING

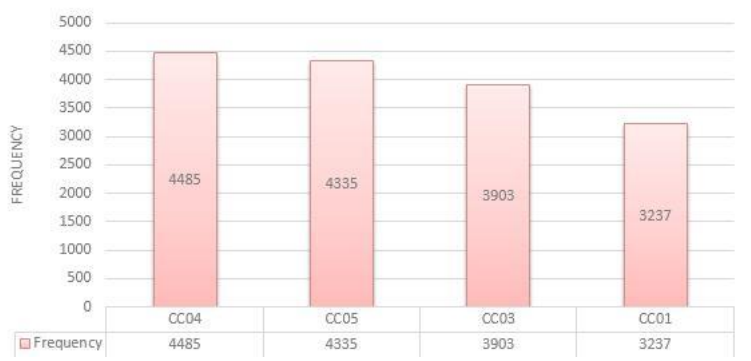

Figure 12. Resources frequency after deleted delivery cancel loading

From the tables and graphs, it can be seen that the tool that is often used is $\mathrm{CC} 04$ with an average duration of 21 hour 36 minutes with a frequency of use of 4485 times.

By reducing the frequency of occurrence of cancel loading delivery events and even by eliminating, because then the time needed for the loading and unloading cycle will be reduced, making it more effective and efficient. Next, a comparison before and after the cancel loading delivery activity will be displayed, so that the amount of time savings can be determined directly.

TABLE 20.

COMPARISON BEFORE AND AFTER ELIMINATION OF THE CANCEL LOADING DELIVERY PROCESS

\begin{tabular}{lccc}
\hline \hline \multicolumn{1}{c}{ Comparison } & Before & After & Difference \\
\hline Median case duration & 17,7 hours & 17,4 hours & 0,3 hours \\
Mean case duration & 65,4 hours & 53,8 hours & 11,6 hours \\
Number of Variants & 279 & 255 & 24 \\
\hline \hline
\end{tabular}

From the table above, it can be seen that after the elimination process in cancel delivery activities is carried out, the average duration of time is shorter, so it is more efficient and can improve the performance of the flow of container activity processes. The difference in average time is 11.6 hours. This value is very influential on the flow of container loading and unloading processes, by saving time it can reduce the number of ship queues and facilitate container loading and unloading operations.

\section{CONCLUSION}

In conclusion, after eliminating the cancel delivery activities, the reduction in the duration of the average time from 65.4 hours to 53.8 hours is reduced. So, the time decreases by \pm 11.6 hours, which means it reduces the cycle time and increases more productivity of the loadingunloading container.

\section{REFERENCES}

Use the "Insert Citation" button to add citations to this document. 